\title{
A Novel Degradation Estimation Method for a Hybrid Energy Storage System Consisting of Battery and Double-Layer Capacitor
}

\author{
Yuanbin Yu, ${ }^{1}$ Dongdong Zhang, ${ }^{1}$ Haitao Min, ${ }^{1}$ Yi Tang, ${ }^{2}$ and Tao Zhu ${ }^{1}$ \\ ${ }^{1}$ State Key Laboratory of Automotive Simulation and Control, Jilin University, Changchun 130022, China \\ ${ }^{2}$ Continental Automotive Corporation (LYG) Co., Ltd., Changchun Branch, Changchun 130022, China \\ Correspondence should be addressed to Haitao Min; minht@jlu.edu.cn
}

Received 30 December 2015; Revised 18 February 2016; Accepted 24 March 2016

Academic Editor: Ruben Specogna

Copyright (C) 2016 Yuanbin Yu et al. This is an open access article distributed under the Creative Commons Attribution License, which permits unrestricted use, distribution, and reproduction in any medium, provided the original work is properly cited.

\begin{abstract}
This paper presents a new method for battery degradation estimation using a power-energy (PE) function in a battery/ultracapacitor hybrid energy storage system (HESS), and the integrated optimization which concerns both parameters matching and control for HESS has been done as well. A semiactive topology of HESS with double-layer capacitor (EDLC) coupled directly with DC-link is adopted for a hybrid electric city bus (HECB). In the purpose of presenting the quantitative relationship between system parameters and battery serving life, the data during a 37-minute driving cycle has been collected and decomposed into discharging/charging fragments firstly, and then the optimal control strategy which is supposed to maximally use the available EDLC energy is presented to decompose the power between battery and EDLC. Furthermore, based on a battery degradation model, the conversion of power demand by PE function and PE matrix is applied to evaluate the relationship between the available energy stored in HESS and the serving life of battery pack. Therefore, according to the approach which could decouple parameters matching and optimal control of the HESS, the process of battery degradation and its serving life estimation for HESS has been summed up.
\end{abstract}

\section{Introduction}

Li-ion battery and EDLC are widely used as energy storage systems to improve fuel economy of hybrid electric vehicle (HEV) [1, 2]. Hybrid energy storage system (HESS) could combine the advantages of enormous energy in battery and large power as well as long life cycle in EDLC, respectively $[3,4]$; meanwhile it also has the potentials in reducing loss and regenerating energy even in critical ambient $[2,5-7]$. In actual operation, the vehicle will work in exhaustive ways, and the HESS will experience frequently switching between charging and discharging. To a certain extent, there has been comprehension of the influence of battery degradation [8-10] and the test results have shown that the battery serving life depends on the discharge depth of each cycle and the cumulative energies during operating, under a certain temperature. But in terms of HESS used in vehicles, it seems that there are no rational methods or processes which can dispose a large number of data of statistics and analysis on power and energy, with less time consumed.

In our previous study [11, 12], the concept power-energy (PE) function has been devised to illustrate the requirements of power demand and capability of HESS. By means of mathematical transformation, the PE function could be standardized in the form of PE matrix and applied to the design process [11]. In [12], the concept of the PE function has also been used into multiobjectives optimization of a semiactive HESS for a HEV, and the optimization which can decrease system mass, volume, and cost has been completed, and the minimum matched line for HESS has also been obtained. But the issue of how to optimally control the HESS and how long the expected battery life is remains to be further studied.

Overall, in terms of performance requirements and complexity, the integrated optimization of parameters matching and control of HESS still needs to be further studied, especially for the battery degradation estimation, which is applied 
in proposed vehicles [13-16]. In this paper, the semiactive topology of HESS with EDLC directly coupled with DC-link is adopted for a hybrid electric city bus (HECB); the powerenergy (PE) function approach is utilized to deal with the integrated optimization for deriving the optimal matching and control of the HESS, with battery degradation taken into consideration. The objectives of this paper are as follows:

(1) Present feasible statistics and analysis method of battery energy flowing during a driving cycle. The data during a driving cycle have been collected and decomposed into discharging/charging fragments firstly; then the optimal power allocation strategy which is supposed to maximize the use of available EDLC energy is presented to decompose the power allocation between battery and EDLC.

(2) Provide an approach to establish the relationship between battery available energy and battery serving life. Based on a battery degradation model which has been proposed in [12], the conversion of power demand by PE function and PE matrix is applied to evaluate the relationship between the available energy stored in HESS and the serving life of battery.

(3) Sum up the process of battery serving life estimation in HESS. According to the approach which can decouple parameters matching and optimal control of the HESS, the battery degradation and its serving life estimation process for the HESS proposed in this paper has been summed up.

The research route of this paper is as follows: the data during a 37-minute driving cycle have been collected and decomposed into discharging/charging fragments of working modes firstly; then in order to decouple the integrated optimization problem of parameters matching and control, the optimal control strategy is presented which is supposed to be able to maximize the use of available EDLC energy, and, at the same time, the decomposition of power between batteries and EDLC is obtained. Furthermore, based on a battery degradation model, the conversion of power demand by $\mathrm{PE}$ function and PE matrix is applied to establish the relationship between the available energy stored in HESS and the serving life of battery.

\section{Working Modes Definition and Optimal Power Allocation Strategy of HESS}

2.1. Working Modes Definition of HESS. A series-parallel HECB which comprises of EDLC, battery, DC/DC converter, engine, integrated starter/generator (ISG), clutch, electric motor (EM), and transmission is used as a case studied in this paper and has been shown in Figure 1. The specifications of the HECB can be seen in [12]. Accessories such as air conditioning and vacuum booster system are electrically driven. In order to make full use of the advantages in terms of EDLC efficiency and life, the EDLC is placed at both ends of DC-link, and inverters are responsible for converting the DC power into three-phase electric for EM/ISG; the input/output powers of the battery are controlled by DC/DC converter.

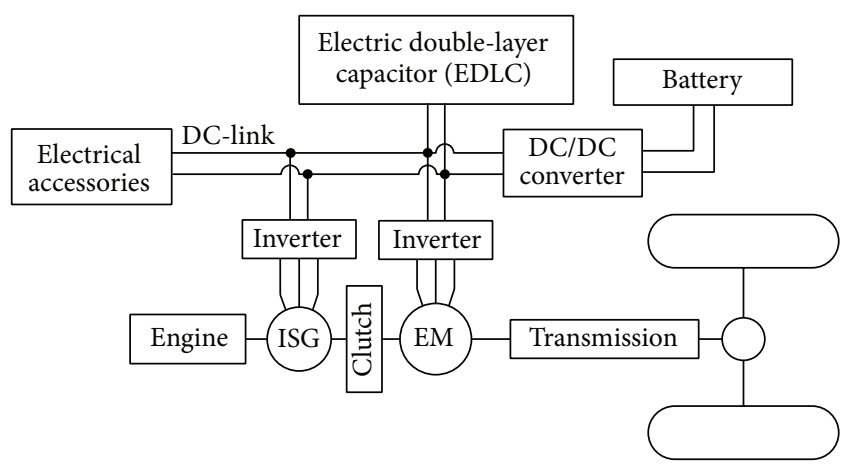

FIgURE 1: Configuration of the hybrid electric city bus (HECB).

Operating data of a 37-minute driving cycle in Changchun China have been collected and shown in Figure 2; also it can be seen that the HESS on the bus will switch frequently between charging and discharging. To gain a deep understanding of the power and energy requirement for HESS, a fragment of electric power requirements is presented in Figure 3. In order to facilitate the statistics of vast data during driving cycles, the working modes have been defined into charging, idle, and discharging modes, corresponding to the electric power requirements of HESS. According to the definition shown in Figure 3, the 37-minute Changchun cycle shown in Figure 2 contains 59 discharging and 73 charging fragments.

In [11], the PE function method has been defined to present the relationship between power and energy of HESS, which can illustrate the capability of HESS and the power demand in the same chart and analyze the battery/EDLC characteristics' effect on the performance of HESS more intuitively. The nondecreasing convex character of PE function has also been derived in [11]. In PE function, shown by (1), the independent variable $P$ (in $\mathrm{kW}$ ) is defined as a threshold of power demand, and the dependent variable $E$ (in Wh) represents the part of energy required by the power demand that exceeds $P$ :

$$
\begin{aligned}
E & =F(P), \\
F(P) & =\int_{0}^{T} f(t) d t, \quad f(t)= \begin{cases}0, & P(t)<P, \\
P(t)-P, & P(t) \geq P .\end{cases}
\end{aligned}
$$

2.2. Battery Degradation Model. According to [8-10], degradation of battery represented by the percentage of capacity loss/initial capacity mainly depends on the cumulative electric quantity provided by battery at certain operating temperature, and the relationship between degradation of battery and the cumulative electric quantity during charging and discharging can be shown as

$$
\mathrm{Q}_{\text {loss }}=K_{\text {life }}\left(\mathrm{Ah}_{\text {through }}\right)^{Z} \text {, }
$$

where $Q_{\text {loss }}$ is the percentage of capacity loss/initial capacity and hereinafter represents the degradation of battery. $\mathrm{Ah}_{\text {through }}$ is the integral of current-time during charging/discharging and hereinafter represents the cumulative 


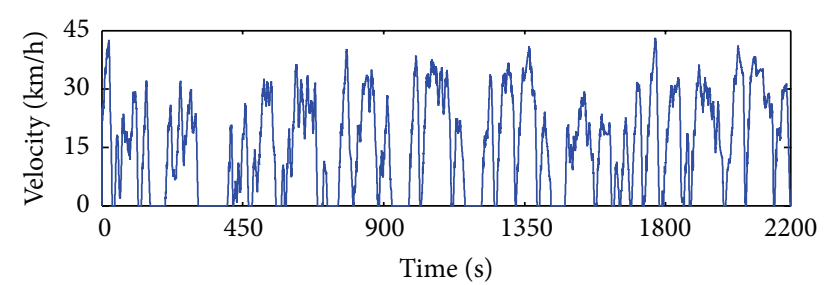

FIgURE 2: The 37-minute driving cycle in Changchun.

electric quantity provided by battery. $K_{\text {life }}$ is life coefficient which is related to the characteristics of battery, and $Z$ is the exponential based on battery characteristics, of which the value is constant 0.55 .

For battery used in HEV, its state of charge (SOC) is expected to be controlled at the midpoint of $50 \%$ and able to float up and down by $10 \%$, to ensure that the battery has a long life performance [1]. According to the charging/discharging characteristics of the battery, within the $40 \% \sim 60 \%$ SOC range, the open circuit voltage (OCV) of the battery can be approximately considered as a constant [2]. So the following equation can be established:

$$
E_{\mathrm{BAT}}=C_{\mathrm{BAT}} \times \mathrm{OCV} \times(0.6-0.4) \times 50 \%,
$$

where $E_{\mathrm{BAT}}$ is the available battery energy for charging/discharging (Wh). OCV is the open circuit voltage of the battery $(\mathrm{V}) . \mathrm{C}_{\mathrm{BAT}}$ is the battery capacity (Ah).

With the assumption that the battery SOC is always floating up and down $10 \%$ from the midpoint, the relationship between the cumulative energy during charging/discharging and $\mathrm{Ah}_{\text {through }}$ can be established as follows:

$$
\mathrm{Ah}_{\text {through }}=\frac{\mathrm{Wh} \text { through }}{\mathrm{OCV}}=\frac{\mathrm{Wh}}{10 \times E_{\mathrm{BAT}}},
$$

where $\mathrm{Wh}_{\text {through }}$ is the cumulative energy during charging/discharging (Wh).

Bringing (4) into (2), the relationship between $E_{\mathrm{BAT}}$ and $Q_{\text {loss }}$ can be established, shown as

$$
Q_{\text {loss }}=K_{\text {life }}\left(\frac{\mathrm{Wh}_{\text {through }} \times C_{\mathrm{BAT}}}{10 \times E_{\mathrm{BAT}}}\right)^{0.55} .
$$

Using (5), when the battery capacity $\left(C_{\mathrm{BAT}}\right)$ has been chosen, the degradation of battery at any $E_{\mathrm{BAT}}$ can be estimated according to the cumulative energy provided by battery.

\subsection{Optimal Power Allocation Strategy of HESS. As men-} tioned in [17-19], for the configuration studied in this paper, in order to maximize the use of the available energy stored in the EDLC to improve the system's efficiency, the optimal power allocation strategy of HESS should be as follows: if the total energy demand is less than that stored in the EDLC, the EDLC provides all the power and battery is not involved in operating. On the contrary, if the total energy demand is larger than that stored in the EDLC, in order to guarantee the maximum power limit of the battery, the power allocation method can be used as follows: When the power is less than a certain threshold, whose value is $p$, all the power should be provided by battery. When the power is larger than $p$, the part of the power demand over $p$ should be provided by EDLC. In this way, the energy required from EDLC will decline with the increase of $p$. When the energy required from EDLC is exactly equal to the available energy stored in EDLC, then the power allocation strategy will satisfy the optimal condition which is the optimal allocation strategy, and the power $p$ denoted as $P_{\text {EDLC_threshold }}$ is the threshold of optimal allocation of HESS. The optimal allocation strategy will be used in the following section for energy statistics.

According to the optimal power allocation strategy mentioned above, if the available energy stored in EDLC has been known beforehand, the threshold of $P_{\text {EDLC_threshold }}$ can be calculated by PE function, and the power of the battery and EDLC can be separated conveniently. According to the definition of $\mathrm{PE}$ function, the value $F\left(P_{\text {EDLC_threshold }}\right)$ is the energy required, corresponding to the extra power demand that exceeds $P_{\text {EDLC_threshold }}$, which should be equal to the available energy stored in EDLC $\left(E_{\text {EDLC }}\right)$. $P_{\text {EDLC_threshold }}$ should satisfy the condition shown as

$$
E_{\text {EDLC }}=F\left(P_{\text {EDLC_threshold }}\right) \text {. }
$$

With a certain power demand, the energy provided by battery is equal to the energy which equals the total energy required subtracting the energy provided by EDLC. In PE function, the total required energy corresponding to the power demand and the energy provided by EDLC is $F(0)$ and $F\left(P_{\text {EDLC_threshold }}\right)$, respectively; so the energy provided by battery can be calculated as

$$
E=F(0)-F\left(P_{\text {EDLC_threshold }}\right) \text {. }
$$

Under optimal power allocation strategy, energy provided by battery during driving cycle can be written as

$$
E=F(0)-E_{\text {EDLC }} \text {. }
$$

Therefore, the degradation of battery shown in (5) can be written as

$$
\begin{aligned}
& Q_{\text {loss }} \\
& = \begin{cases}K_{\text {life }}\left(\frac{\left(F(0)-E_{\mathrm{EDLC}}\right) \times C_{\mathrm{BAT}}}{10 \times E_{\mathrm{BAT}}}\right)^{0.55}, & F(0) \geq E_{\mathrm{EDLC}}, \\
0, & F(0)<E_{\mathrm{EDLC}} .\end{cases}
\end{aligned}
$$

\section{PE Function Based on Battery Degradation during Driving Cycle}

Through the derivation mentioned in the last section, the degradation of battery during a charging/discharging stage under certain power demand can be estimated by using PE function. However, in the actual operation, the HESS will switch frequently between charging and discharging, resulting in large amount of data. It is not convenient to analyze the data one by one only using PE function, so the PE matrix which has already been proposed in [11] is put into use in this section. By standardizing the PE function deduced by power demand in operating, the mass data and servicing life of battery could be dealt with conveniently. 


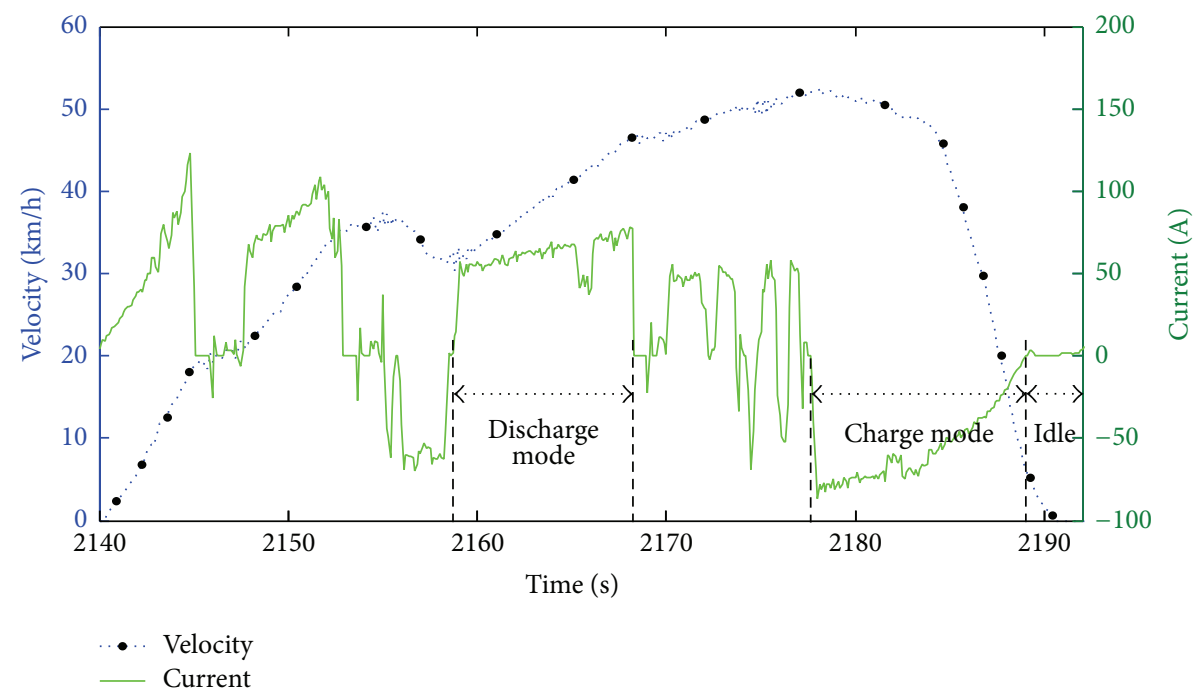

FIGURE 3: Definition of working modes for HESS.

3.1. PE Matrix. In terms of the 59 times of discharging and 73 times of charging described in Section 2.1, the required energy is less than $800 \mathrm{~kJ}$ and the power demand is no more than $90 \mathrm{~kW}$. PE matrix $Q$ is then defined as an $800 * 90$ Boolean matrix. As for a certain fragment of the power demand, the value of each element in its PE matrix is determined as

$$
Q_{m, n}=\left\{\begin{array}{ll}
0, & F(n) \leq m, \\
1, & F(n)>m,
\end{array} \quad m=0: 799, \quad n=0: 89,\right.
$$

where $F(n)$ is the PE function value of the power demand in a certain fragment, $m$ is the row number of the matrix corresponding to the axis of energy in its PE function (unit: $\mathrm{kJ}$ ), and $n$ is the column number of the matrix corresponding to the axis of power in its PE function (unit: $\mathrm{kW}$ ). The elements are then ordered according to the coordinates of the $\mathrm{PE}$ functions, as shown in the following:

$$
Q=\left(\begin{array}{ccc}
Q_{799,0} & \cdots & Q_{799,89} \\
\vdots & \ddots & \vdots \\
Q_{0,0} & \cdots & Q_{0,89}
\end{array}\right)
$$

By adopting PE matrix, the useful information of power demand during driving cycles can be accessed and recorded conveniently, particularly for data processing in microcontroller unit. PE matrix is not only convenient for data statistics, but also easier to use computer to interpret and process data. According to the definition of PE function, $\mathrm{PE}$ matrix $Q$ has the following four properties:

(i) The required energy for a certain fragment of power demand $\left(E_{\text {required }}\right)$ is the sum of the column elements whose column number is 0 in the PE matrix:

$$
E_{\text {required }}=\operatorname{sum}\left(Q_{0: 799,0}\right) \text {. }
$$

(ii) If the power limit of battery is $P_{\text {BATlimit }}$, the available energy of EDLC $\left(E_{\mathrm{EDLC}}\right)$ must be no less than the sum of column elements whose column number is $P_{\text {BATlimit }}$ in the PE matrix (if $P_{\mathrm{BATlimit}}$ is not an integer, $P_{\mathrm{BATlimit}}$ should value down to take an integer, and then the charge/discharge capacity of HESS can meet the power demand ultimately), as shown below:

$$
E_{\mathrm{EDLC}} \geq \operatorname{sum}\left(Q_{0: 799, P_{\mathrm{BATlimit}}}\right)
$$

(iii) If the available energy of EDLC is $E_{\mathrm{EDLC}}$, the threshold of optimal allocation at that power demand $P_{\text {EDLC_threshold }}$ is the sum of row elements whose row number is $E_{\mathrm{EDLC}}$ in the PE matrix (if $E_{\mathrm{EDLC}}$ is not an integer, $E_{\mathrm{EDLC}}$ should value down to take an integer). Only when the power limit $P_{\text {BATlimit }}$ is not smaller than $P_{\text {EDLC_threshold }}$, the charge and discharge capacity of HESS can meet the power demand ultimately, as shown in the following:

$$
P_{\text {EDLC_threshold }}=\operatorname{sum}\left(Q_{E_{\mathrm{EDLC}}, 0: 89}\right) \text {. }
$$

(iv) The energy provided by the battery during the driving cycle can be written as

$$
E=\operatorname{sum}\left(Q_{E_{\mathrm{EDLC}}: 799,0}\right)
$$

Using PE matrix, the estimation of degradation shown in (9) during a certain driving cycle can be written as

$$
Q_{\text {loss }}=K_{\text {life }}\left(\frac{\operatorname{sum}\left(Q_{E_{\mathrm{EDLC}}: 799,0}\right) \times C_{\mathrm{BAT}}}{10 \times E_{\mathrm{BAT}}}\right)^{0.55} \text {. }
$$

3.2. PE Density Matrix. By using the PE matrix, the PE function is standardized, which can be used to store, process, and analyze a large amount of power demand information in the form of PE matrix to estimate the battery life of HESS. In the 37-minute running data of the hybrid electric vehicle, 59 times discharging working fragments and 73 times charging working fragments are included. A new matrix $W$ is obtained 


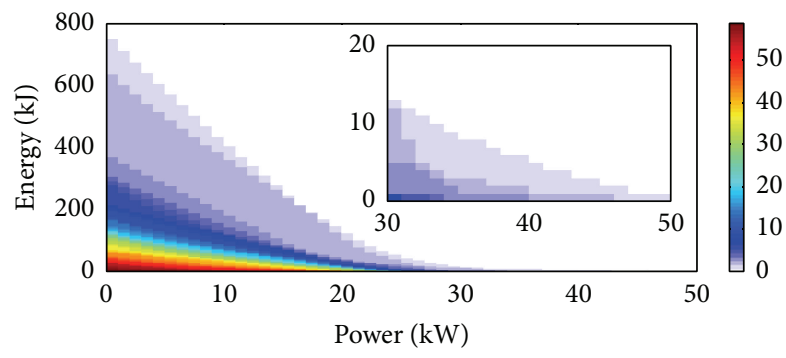

FIgURE 4: The PE density matrix in discharging fragments.

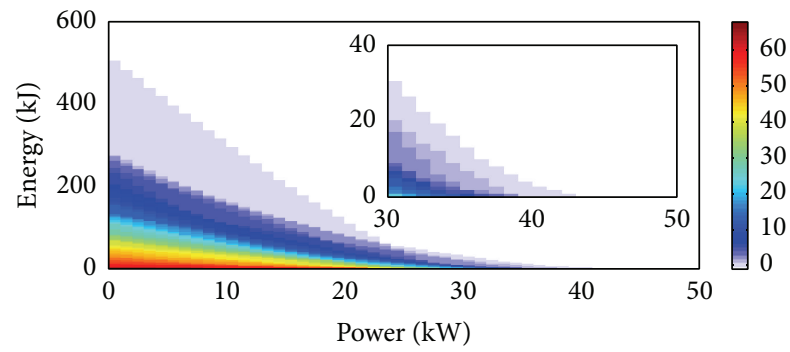

FIGURE 5: The PE density matrix in charging fragments.

by adding the power demand PE matrix of the 59 times discharging work fragments to a new matrix, which is called the PE density matrix, shown as

$$
W=\left(\begin{array}{ccc}
W_{799,0} & \cdots & W_{799,89} \\
\vdots & \ddots & \vdots \\
W_{0,0} & \cdots & W_{0,89}
\end{array}\right)
$$

The matrix has the same row and column numbers as the PE matrix, and the value of $W_{m, n}$ is the sum of corresponding elements in the PE matrix of the power demand in the 59 times discharging working fragments $\left(Q_{m, n}\right)$. The PE density matrix contains the whole discharging power demand information of the HESS during cycling, as shown in Figure 4.

As shown in Figure 4, this paper uses the PE density matrix in the same method as the PE matrix and uses different colors to represent the values of $W_{m, N}$ in different PE density matrix. Similarly, the PE matrix of the power demand in the 73 times charging working fragments is added, and the whole charging power demand information of the HESS during cycling can be obtained, as shown in Figure 5.

By adding the corresponding PE matrix in the 73 times charging and 59 times discharging fragments, the PE density matrix which can represent the power demand information on behalf of the entire duration of 37 minutes cycle can be obtained, as shown in Figure 6.

During the cycle, the battery degradation is the sum of that in each operation fragments. According to the optimal power allocation strategy, $P_{\text {EDLC_threshold }}$ is determined by the power demand and the EDLC available energy $\left(E_{\text {EDLC }}\right)$ and the power demand in each operation fragment, which means that each operation fragment will correspond to an optimal allocation power value. Fortunately, from the relationship shown in (16), the battery degradation does not depend on

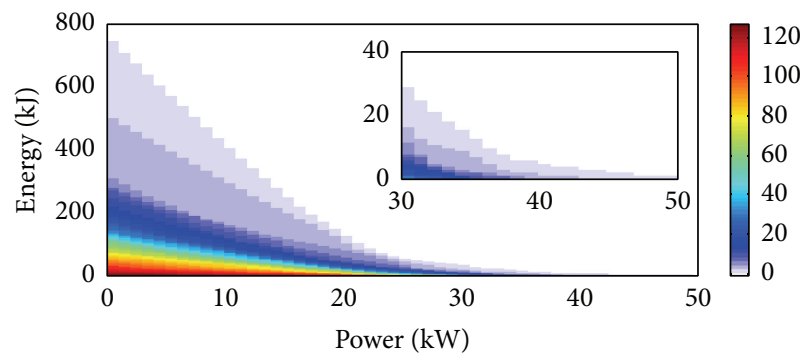

FIGURE 6: The PE density matrix during the whole cycle.

the value of $P_{\text {EDLC_threshold }}$. Namely, in (16), the PE matrix $Q$ can be alternative to the PE density matrix $W$, and the battery degradation during the whole cycle can be shown as follows:

$$
Q_{\text {loss }}=K_{\text {life }}\left(\frac{\operatorname{sum}\left(W_{E_{\mathrm{EDLC}: 799,0}}\right) \times C_{\mathrm{BAT}_{\mathrm{B}} \mathrm{C}}}{10 \times E_{\mathrm{BAT}}}\right)^{0.55} .
$$

\section{Battery Serving Life Estimation}

4.1. Cycling Life Estimation of Battery. In (18), the charging/discharging cumulative energy of battery degradation can be calculated. The available energy of battery $\left(E_{\mathrm{BAT}}\right)$ is reduced to the lowest (denoted as $E_{\text {BATlow }}$ ) but still can satisfy the charging/discharging demand during battery servicing life; then at the end of battery servicing life, the degradation of battery $Q_{\text {loss }}$ can be expressed as

$$
\mathrm{Q}_{\mathrm{loss}}=\frac{E_{\mathrm{BAT}}-E_{\mathrm{BATlow}}}{E_{\mathrm{BAT}}} \times 100 \% \text {, }
$$

where $E_{\mathrm{BATlow}}$ is the lowest available energy of battery to satisfy charging/discharging demand when the HESS matched in $\left(E_{\mathrm{BAT}}, E_{\mathrm{EDLC}}\right)$. The anticipated driving cycles $\left(N_{\text {life }}\right)$ during the whole battery cycling life can be calculated in

$$
\begin{aligned}
& \frac{E_{\mathrm{BAT}}-E_{\text {BATlow }}}{E_{\mathrm{BAT}}} \times 100 \\
& =K_{\text {life }}\left(\frac{N_{\text {life }} \times \operatorname{sum}\left(W_{E_{\mathrm{EDLC}}: 799,0}\right) \times C_{\mathrm{BAT}}}{10 \times E_{\mathrm{BAT}}}\right)^{0.55} .
\end{aligned}
$$

According to [8], $K_{\text {life }}$ is determined by the physical characteristics and the temperature of battery when charging/discharging. In this paper, the capacity of the battery cell is $8 \mathrm{Ah}$, and the anticipated degradation is no more than $20 \%$ after 2000 cycles of charging/discharging under $80 \%$ depth of discharge (DOD) at 20 Celsius; then the life coefficient $K_{\text {life }}$ can be calculated to 0.0752 .

By using (20), $N_{\text {life }}$ with different parameter configurations can be calculated as shown in Figure 8. The values of $N_{\text {life }}$ are shown in the form of a contour, and the unit is ten thousand times. As shown in Figure 7, for parameter configurations of HESS, both increasing $E_{\mathrm{EDLC}}$ to reduce the use of battery and increasing $E_{\mathrm{BAT}}$ to increase the allowed battery degradation can all prolong the cycling life of battery, but, from the cost aspect, increasing $E_{\mathrm{EDLC}}$ is more effective. 


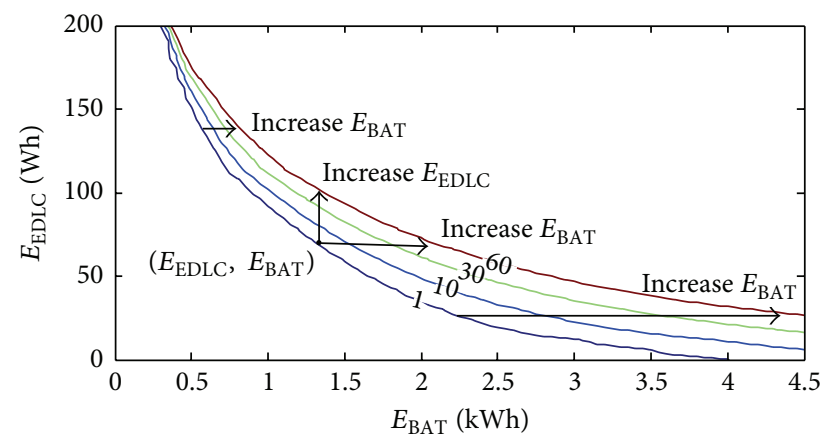

Figure 7: Contour cycles of battery (unit: ten thousand times).

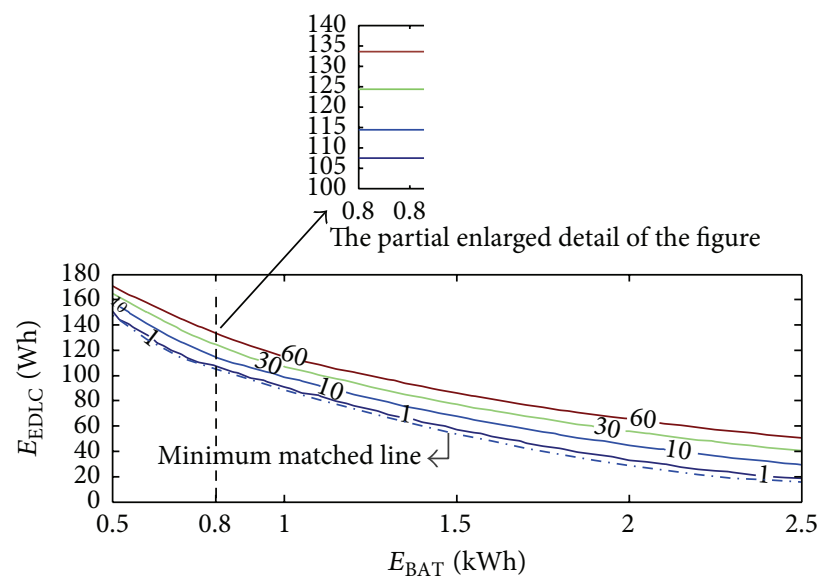

FIGURE 8: Contours of battery service life in vehicular application (unit: years).

According to the data from the $X$ axis, when using battery only, it needs to expand much $E_{\mathrm{BAT}}$ to reach 100000 or 300000 battery cycles. After adding EDLC, the $E_{\mathrm{BAT}}$ increased to reach the battery cycles can be reduced significantly.

4.2. Serving Life Calculation of Battery in Vehicle Application. For the HEV studied in this paper, the average daily operating time of each HEV is about 12 hours. Under the 37-minute city driving cycle, the years that the vehicle can run during battery serving life can be calculated as

$$
\text { Duration }_{\text {life }}=\frac{N_{\text {life }}}{365 \times(12 \times 60 \mathrm{~min}) / 37 \mathrm{~min}},
$$

where Duration life $_{\text {ife }}$ is the years of battery serving life.

Based on (21) and the PE density matrix shown in Figure 6, the battery serving life during cycles in vehicle application can be calculated as shown in Figure 8, and the minimum matched line deduced in [12] is also shown in Figure 8. The results show that, according to the configuration of HESS and the vehicular application requirements mentioned in this paper, when the parameters matching for the available energy of battery and EDLC have been chosen in the coordinate range as shown in Figure 8, the contours of battery serving life in vehicular application are all over the minimum matched line, which has been deduced in [12], and it indicates that the work done in this paper can be used as a reasonable complement to the existing HESS parameters matching. By using the specific methods and configurations proposed in this paper, under the most ideal circumstance, it seems that battery serving life could be as long as 60 years. If available battery energy is still chosen at $0.8 \mathrm{kWh}$ in accordance with [12], in order to meet the needs of 1-60 years battery serving life, the corresponding EDLC available energy should be 107, 114, 124, and $133 \mathrm{Wh}$, respectively.

In summary, by using PE function and its density matrix, if the battery degradation calculation has been obtained in advance, as described in (20), the procedure of battery serving life estimation can be summed up as Figure 9.

\section{Conclusion}

A semiactive topology of HESS with EDLC directly coupled with DC-link is adopted for a hybrid electric city bus in this paper. A power-energy (PE) function is utilized to establish relationship and dispose the integrated optimizations between parameters matching and control of a certain battery/EDLC HESS. The feasible statistics and analysis method of battery energy flowing during driving cycles are presented, and a method to establish the relationship between available battery energy and battery cycling life is provided. Therefore, with the approach of decoupling parameters matching and optimal control, the battery degradation and its cycling life estimation process for the HESS proposed in this paper has been summed up. The estimation process is generally divided into the following 4 steps, which are cycle segment, power allocation, statistics, and estimation. With the application of the above 4 steps, the integrated optimization which concerns both parameters matching and control for HESS could be done as well. The process is also feasible for driving cycle with longer operating time and more charging/discharging fragments. With the increasing of data to be processed, it will be difficult to analyze the data one by one only by PE function. Then the PE matrix and its density matrix would provide great assistance in data processing after power allocation of HESS by PE function.

In general, we try to find an optimal allocation strategy to utilize the charging/discharging capability of EDLC in order to improve the efficiency of HESS; meanwhile the different resistance between battery and EDLC is also taken into account, as well as the DC/DC efficiency. In terms of whole vehicle's control, there are still some other factors, such as EM/ISG efficiency, that should be considered. How to put the optimal power allocation strategy of HESS into real-time control remains to be studied in future research.

\section{Competing Interests}

The authors declare that there are no competing interests regarding the publication of this paper.

\section{Acknowledgments}

The authors gratefully acknowledge the financial support from the National Natural Science Foundation of China 


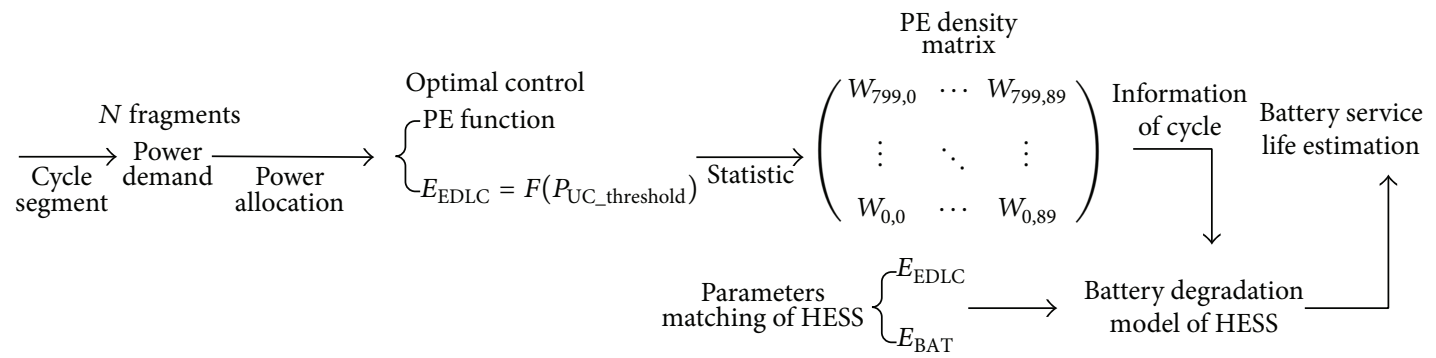

FIGURE 9: The procedure of battery serving life based on PE approach.

(51107052) and the Key Science and Technology Projects of Science and Technology Department of Jilin Province (20150204016GX).

\section{References}

[1] M. A. Hannan, F. A. Azidin, and A. Mohamed, "Hybrid electric vehicles and their challenges: a review," Renewable and Sustainable Energy Reviews, vol. 29, pp. 135-150, 2014.

[2] B. Vulturescu, R. Trigui, R. Lallemand, and G. Coquery, "Implementation and test of a hybrid storage system on an electric urban bus," Transportation Research Part C: Emerging Technologies, vol. 30, pp. 55-66, 2013.

[3] J. Van Mierlo, P. Van den Bossche, and G. Maggetto, "Models of energy sources for EV and HEV: fuel cells, batteries, ultracapacitors, flywheels and engine-generators," Journal of Power Sources, vol. 128, no. 1, pp. 76-89, 2004.

[4] L. Gao, R. A. Dougal, and S. Liu, "Power enhancement of an actively controlled battery/ultracapacitor hybrid," IEEE Transactions on Power Electronics, vol. 20, no. 1, pp. 236-243, 2005.

[5] M. Ayadi, O. Briat, R. Lallemand et al., "Description of supercapacitor performance degradation rate during thermal cycling under constant voltage ageing test," Microelectronics Reliability, vol. 54, no. 9-10, pp. 1944-1948, 2014.

[6] R. M. Schupbach and J. C. Balda, "Comparing DC-DC converters for power management in hybrid electric vehicles," in Proceedings of the IEEE International Electric Machines and Drives Conference (IEMDC '03), pp. 1369-1374, IEEE, Madison, Wis, USA, June 2003.

[7] A. H. Eghbali, B. Asaei, and P. Nader, "Fuel efficient control strategy, based on battery-ultracapacitor energy storage system, in parallel hybrid electric vehicles," in Proceedings of the IEEE Vehicle Power and Propulsion Conference (VPPC '10), pp. 1-5, IEEE, Lille, France, September 2010.

[8] J. Wang, P. Liu, J. Hicks-Garner et al., "Cycle-life model for graphite- $\mathrm{LiFePO}_{4}$ cells," Journal of Power Sources, vol. 196, no. 8, pp. 3942-3948, 2011.

[9] M. Safari, M. Morcret te, A. Teyssot, and C. Delacourt, "Lifeprediction methods for Lithium-ion batteries derived from a fatigue approach," Journal of The Electrochemical Society, vol. 157, pp. A713-A720, 2010.

[10] S. Fiorenti, J. Guanetti, Y. Guezennec, and S. Onori, "Modeling and experimental validation of a hybridized energy storage system for automotive applications," Journal of Power Sources, vol. 241, pp. 112-120, 2013.

[11] Q. Xiaodong, W. Qingnian, and Y. YuanBin, "Power demand analysis and performance estimation for active-combination energy storage system used in hybrid electric vehicles," IEEE
Transactions on Vehicular Technology, vol. 63, no. 7, pp. 31283136, 2014.

[12] C. Zhang, H. Min, Y. Yu, Q. Wang, and H. Sun, "A new method to optimize semiactive hybrid energy storage system for hybrid electrical vehicle by using pe function," Mathematical Problems in Engineering, vol. 2015, Article ID 457303, 14 pages, 2015.

[13] R. Carter, A. Cruden, and P. J. Hall, "Optimizing for efficiency or battery life in a battery/supercapacitor electric vehicle," IEEE Transactions on Vehicular Technology, vol. 61, no. 4, pp. 1526$1533,2012$.

[14] A. J. Fairweather, D. A. Stone, and M. P. Foster, "Evaluation of UltraBattery ${ }^{\mathrm{TM}}$ performance in comparison with a battery-supercapacitor parallel network," Journal of Power Sources, vol. 226, pp. 191-201, 2013.

[15] J. P. Trovão, P. G. Pereirinha, H. M. Jorge, and C. H. Antunes, "A multi-level energy management system for multi-source electric vehicles-an integrated rule-based meta-heuristic approach," Applied Energy, vol. 105, pp. 304-318, 2013.

[16] J. Furukawa, T. Takada, D. Monma, and L. T. Lam, "Further demonstration of the VRLA-type UltraBattery under mediumHEV duty and development of the flooded-type UltraBattery for micro-HEV applications," Journal of Power Sources, vol. 195, no. 4, pp. 1241-1245, 2010.

[17] R. M. Schupbach, J. C. Balda, M. Zolot, and B. Kramer, “Design methodology of a combined battery-ultracapacitor energy storage unit for vehicle power management," in Proceedings of the IEEE 34th Annual Power Electronics Specialists Conference, pp. 88-93, June 2003.

[18] Y.-H. Hung and C.-H. Wu, "An integrated optimization approach for a hybrid energy system in electric vehicles," Applied Energy, vol. 98, pp. 479-490, 2012.

[19] A. Santucci, A. Sorniotti, and C. Lekakou, "Power split strategies for hybrid energy storage systems for vehicular applications," Journal of Power Sources, vol. 258, pp. 395-407, 2014. 


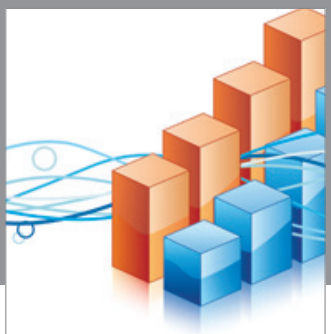

Advances in

Operations Research

vatem alat4

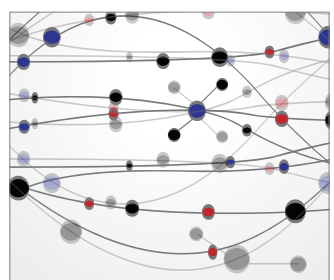

\section{The Scientific} World Journal
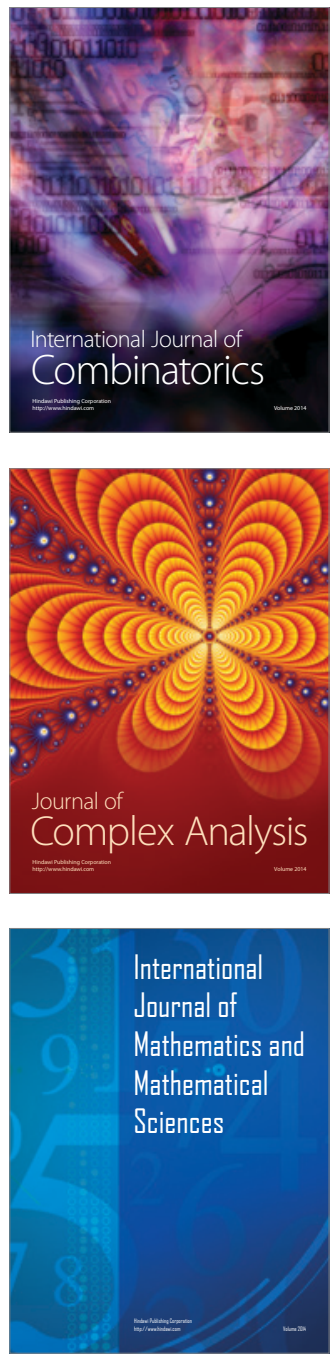
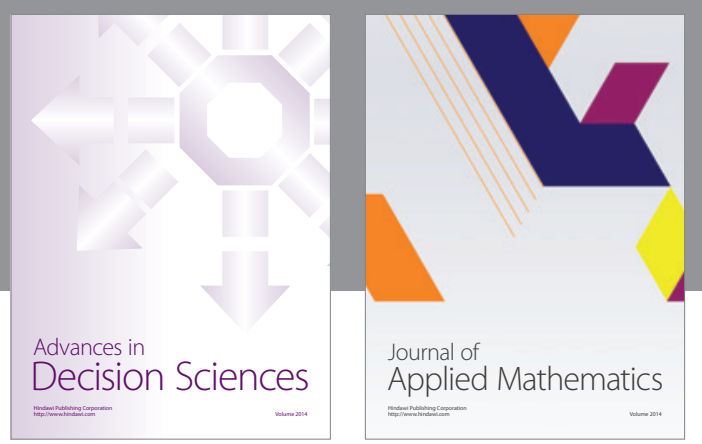

Algebra

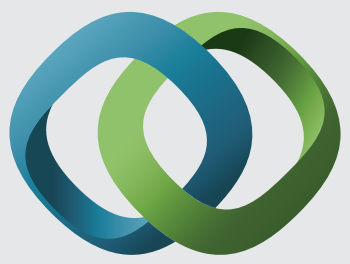

\section{Hindawi}

Submit your manuscripts at

http://www.hindawi.com
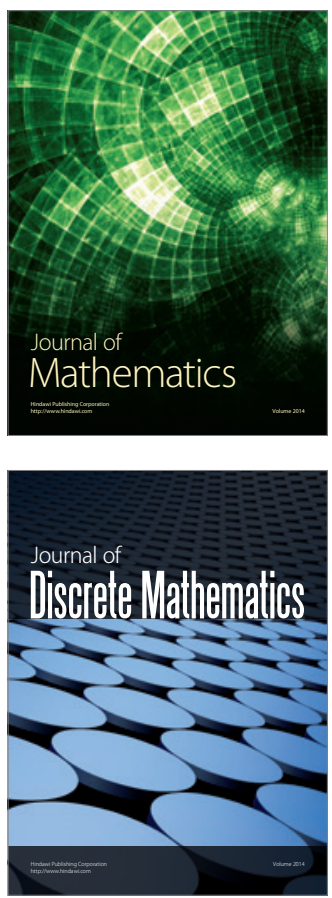

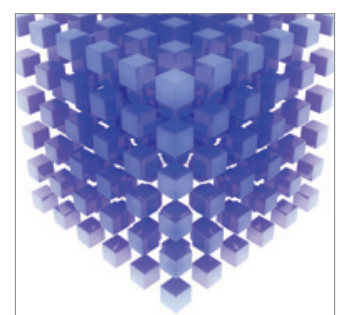

Mathematical Problems in Engineering
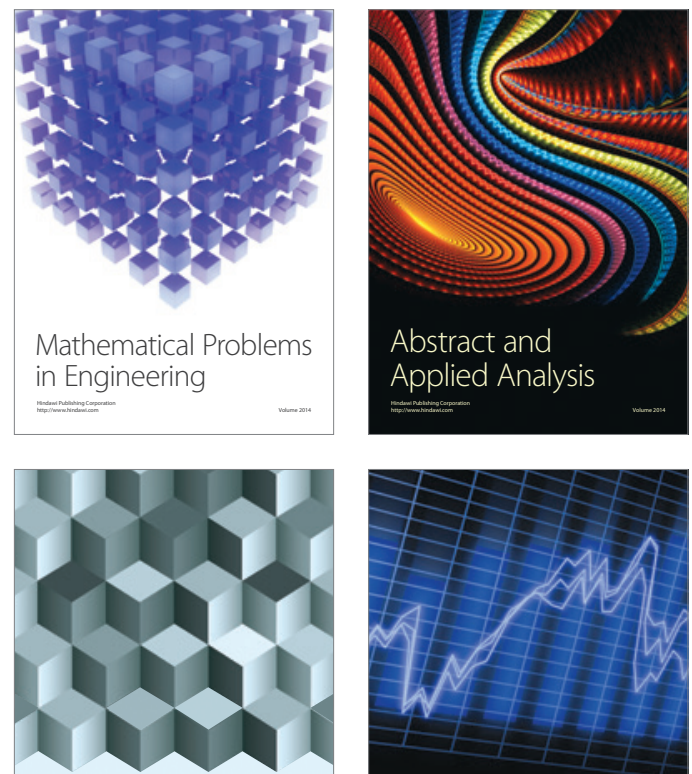

Journal of

Function Spaces

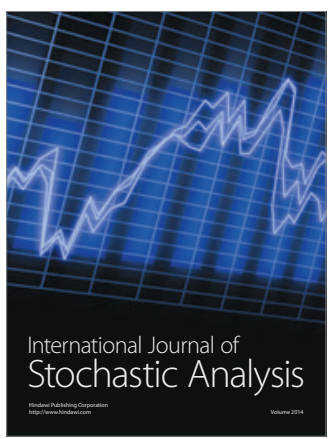

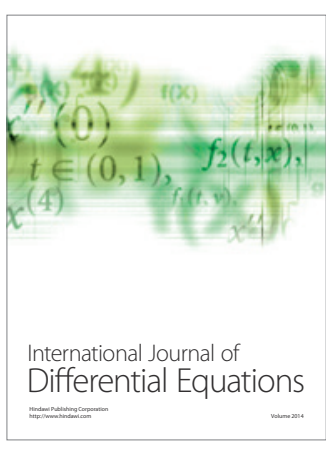
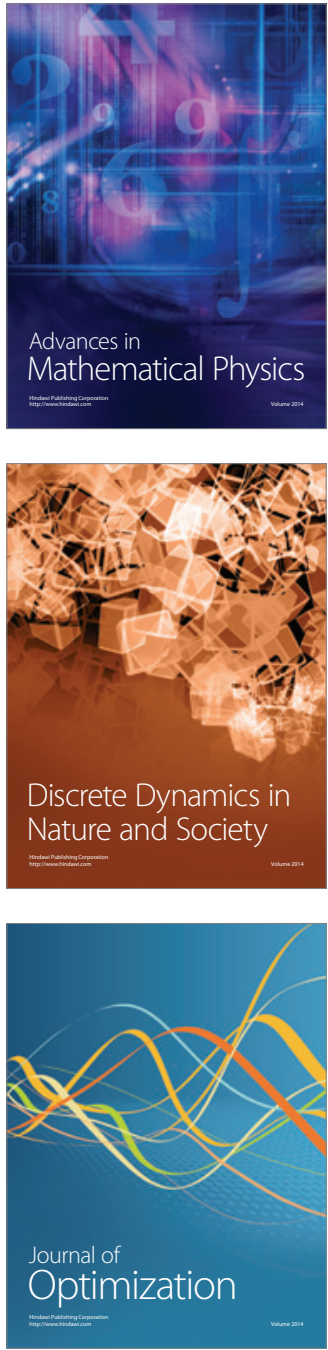